\title{
The model of double-cage induction motor for the analysis of thermal fields in transient operations
}

\author{
JAN MRÓZ \\ Rzeszow University of Technology \\ janmroz@prz.edu.pl
}

(Received: 28.09.2016, revised: 11.02.2017)

\begin{abstract}
Emergency motor switch-on happens occasionally while operating a doublesquirrel-cage motor at full supply voltage with the rotor blocked (e.g., in coal mills). After releasing the blockage, the by now heated motor is started up again. However, the mechanical stress caused by the increased temperature poses considerable hazards to the squirrel-cage winding. This paper presents a double-cage induction motor model for analysis of thermal fields in transient operation. The thermal field for the rotor of a doublesquirrel-cage motor of soldered or cast structure, operating in the conditions described, has been calculated in the present paper using a thermal network method. Measurement results have been presented for the double-squirrel-cage winding temperature for a soldered cage construction in the blocked rotor state.
\end{abstract}

Key words: AC motors, heating, induction motors, squirrel cage motors, temperature rise

\section{Introduction}

As stated in prior research [1,2], while starting up the deep-bar and double-squirrel-cage motors with soldered rotors, the squirrel-cage winding is the component most vulnerable to damage. The method of starting motors through an immediate connection to the rated supply voltage is widely used; however, the risk to the squirrel-cage, resulting from thermal and electro-dynamical action of starting currents, is greatest here. Such phenomena become of extreme importance during the prolonged start-up. Fig. 1 presents a damaged squirrel-cage winding in the end region of the starting cage in a medium-power squirrel-cage motor.

Thermal problems while starting up squirrel-cage motors are referenced in a relatively large number of studies; however, in most cases, the reference is made to deep-bar motors started up with either an immediate connection to a full supply voltage [1] or with a blocked rotor [3]. The heat issues surrounding double-squirrel-cage motors operating in such conditions are represented only in a small number of publications [4]. This paper presents a doublecage motor model with a cast structure rotor and a soldered cage to analyse the thermal field of the rotor in selected, severe operating conditions. Cases in which the motor can be switched on with blocked rotor (mill motor) have been investigated. In such cases, the motor operates 
for several seconds and is switched off. After releasing the blockage, the motor is started up again.

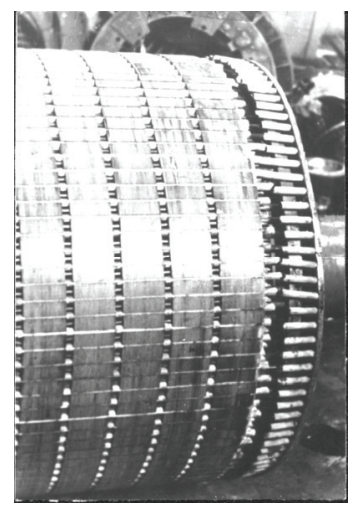

Fig. 1. Damaged end region of the starting cage in a medium-power squirrel-cage motor

\section{Calculating the thermal field of a double-squirrel-cage rotor}

The studied operating states of a double-squirrel-cage-motor are accompanied by considerable variations in the motor thermal field. To analyse such variations, the algorithm presented in Fig. 2 has been adapted.

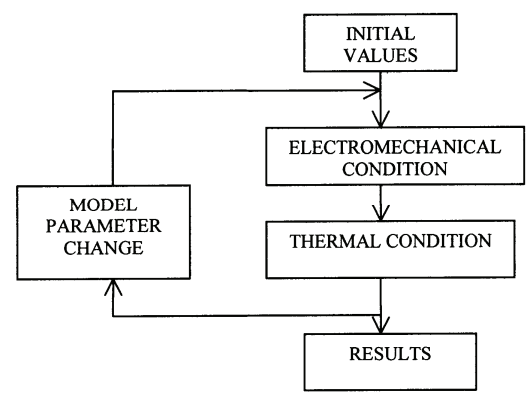

Fig. 2. Algorithm for calculating the rotor thermal field

Once the initial temperature values have been assigned, the electromechanical condition is calculated. As the aperiodic components of the starting current - because of its short persistence compared to that of a prolonged start up - have minor influence on the winding temperature, their actions can be neglected [2]. Moreover, they have little influence on the starting time, as positive oscillations, present at the beginning of the start up, reduce its duration, whereas negative ones prolong it. Consequently, the starting time is almost the same as that calculated from torque static characteristics. Furthermore, it is assumed that the start up takes place in a quasi-static manner, which allows the utilisation of speed sequences and rms current values in winding for calculating the static torque load characteristics and temperature. In each 
stage of calculations, the change (including the temperature) of resistances present in the equivalent motor circuit diagram is taken into consideration.

By determining the motor thermal field in the first step, it becomes possible to change the model parameters, including resistance (effect of the temperature) and reactance (effect of the saturation) of the motor. The software I have designed additionally allows to carry out calculations that take into account changes of bar dimensions (affecting the slot clearance) and changes of material constants due to changes of temperature. The changing slot clearance influences the heat exchange conditions. This option was not used for calculations presented in this paper.

Changing the model parameters is required to initiate the next calculation stage, which is accomplished by repeating the step previously described. In this way, the relationship is considered between the transient electromechanical condition and the transient thermal condition with the accuracy of a single time step. The calculations are completed when the condition defining selected electromechanical state is met (such as the end of start up or when constant speed has been reached).

Prior research [2] has proven that for start up states, the heating of stator components can be examined regardless of the rotor heating because even in prolonged start up conditions, the heat exchange between the rotor and the stator, the body shape, and the ventilating system are of minor importance. Therefore, no heat exchange between stator and rotor is assumed. However, the heat from both rotor and stator surface is transferred to cooling air. Fig. 3 shows the related heat transfer coefficients $\alpha$. Due to the symmetry of construction and load distribution in the double-squirrel-cage motor, the analysed area has been confined in the peripheral direction, as far as the middle of the rotor slot pitch to half of the rotor length. In the radial direction, the analysed area extends from the inner surface of the rotor yoke to the external surface of the rotor pack. Fig. 3 presents part of the double-squirrel-cage rotor with separate end rings of soldered construction (on the left) and a cast cage (on the right).
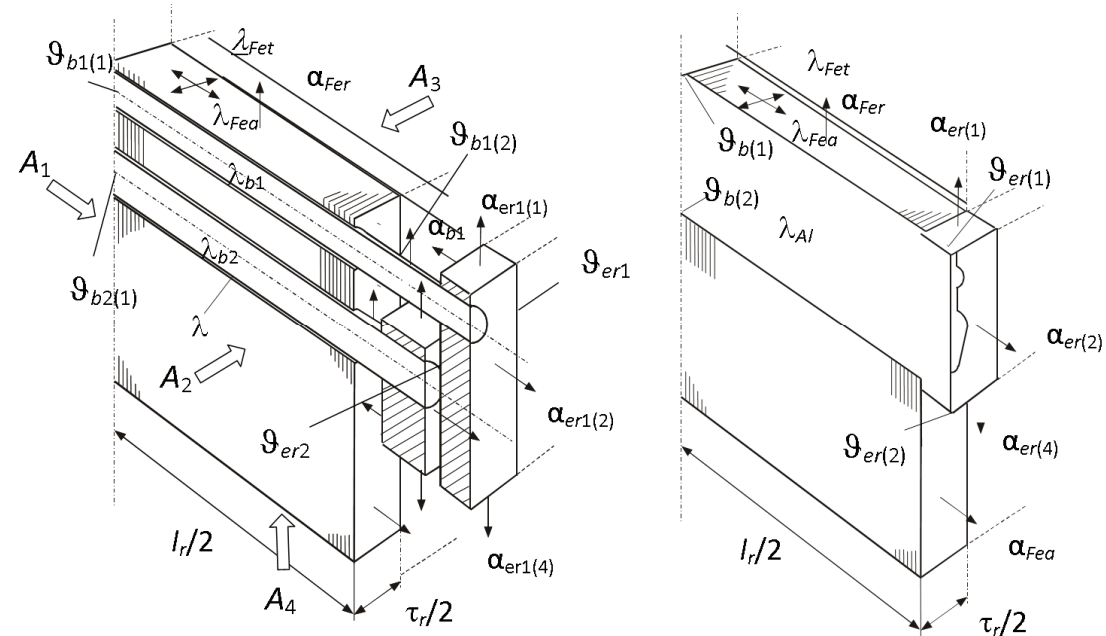

Fig. 3. Diagram of the double-squirrel-cage rotor with separate end rings (on the left), a cast cage (on the right) 
For the cast cage, the end rings come in immediate contact with the rotor core. The symbol $\alpha$ with subscripts denotes the heat transfer coefficient of individual components. The symbol $\lambda$ with respective subscripts is used to denote coefficients of thermal conductivity of respective rotor parts. The symbol $l_{r}$ denotes the length of the rotor core. The symbol $\tau_{r}$ denotes the rotor slot pitch.

To calculate the thermal field of the motor, the thermal network method, created by means of the control volume method, has been utilised [5]. This method involves the division of the object examined into components of any shapes (determined control volumes) and drawing up energy balances for them, which allows the study of heat exchange issues in the areas with complex shapes. It is assumed that the heat capacity of a component focuses on a single node that lies in the component centre of gravity. The fulfilment of the energy balances in separated control volumes allows us to determine, with great accuracy, the temperature distribution and the heat flux density. This method provides obvious physical interpretations, and it involves a larger number of equations than the boundary elements method. However, the matrix is banded and symmetric, which considerably simplifies the solution.

The equation of transient heat conductivity in the area containing inner sources of heat of volume density $q_{V}$ is as follows [5]:

$$
c \rho \frac{\partial \vartheta}{\partial t}=-\operatorname{div} \mathbf{q}+q_{V},
$$

where: $c$ is the specific heat, $\mathbf{q}$ is the heat flux density vector, $\vartheta$ is the temperature, and $\rho$ is the density. The heat does not flow through planes $A_{1}, A_{2}$, and $A_{3}$ (Fig. 3), as they are symmetry planes. The boundary condition of the second type is present:

$$
\lambda \frac{\partial \vartheta}{\partial \mathrm{n}}=q_{A}=0,
$$

where: $q_{A}$ is the surface density of the heat flux and $\lambda$ is the coefficient of thermal conductivity.

In the inner surface of the rotor yoke (plane $A_{4}$ in Fig. 3), which is considerably distant from the essential heat sources, the constant temperature has been assumed and the boundary condition of the first type is present:

$$
\vartheta=\vartheta_{a},
$$

where: $\vartheta_{a}$ is the ambient temperature.

From other surfaces, the heat is transferred with heat transfer coefficients $\alpha$ in Fig. 3, where the boundary condition of the third type occurs:

$$
\lambda \frac{\partial \vartheta}{\partial \mathrm{n}}=\alpha\left(\vartheta_{A}-\vartheta\right),
$$

where: $\vartheta_{A}$ is the fluid temperature. Having integrated Eq. (1) for volume $V_{i}$, based on the Green-Gauss-Ostrogradski theorem for a single element, the following is obtained: 


$$
\int_{V_{i}} c \rho \frac{\partial \vartheta}{\partial t} \mathrm{~d} V=-\int_{A_{i}} \boldsymbol{n} \cdot \boldsymbol{q} \mathrm{d} A+\int_{V_{i}} q_{V} \mathrm{~d} V
$$

where: $\boldsymbol{n}$ is the vector normal to area $A$.

Individual terms of Eq. (5) can be presented as follows:

$$
\begin{gathered}
\int_{V_{i}} c \rho \frac{\partial \vartheta}{\partial t} \mathrm{~d} V \approx V_{i} \rho_{i} c_{i} \frac{\mathrm{d} \vartheta_{i}}{\mathrm{~d} t}, \\
-\int_{A_{i}} \boldsymbol{n} \cdot \boldsymbol{q} \mathrm{d} A=\sum_{j} Q_{j i}+Q_{A i}, \\
\int_{V_{i}} q_{V} \mathrm{~d} V=V_{i} q_{V i},
\end{gathered}
$$

where: $Q_{A i}$ is the heat flux flowing to the node $i$ from the external surface and $Q_{j i}$ is the heat flux flowing from node $j$ to node $i$. Bear in mind in Eq. (7):

$$
\begin{aligned}
Q_{j i} & =\frac{1}{R_{t h i j}}\left(\vartheta_{j}-\vartheta_{i}\right), \\
Q_{A i} & =\frac{1}{R_{t h A i}}\left(\vartheta_{A i}-\vartheta_{i}\right),
\end{aligned}
$$

and for any element $i$, the energy balance can be expressed in the following form:

$$
\sum_{j} \frac{1}{R_{t h i j}} \vartheta_{j}+\frac{1}{R_{t h A i}} \vartheta_{A i}-\left(\frac{1}{R_{t h A i}}+\sum_{j} \frac{1}{R_{t h i j}}\right) \vartheta_{i}+V_{i} q_{V i}=V_{i} c_{i} \rho_{i} \frac{\mathrm{d} \vartheta_{i}}{\mathrm{~d} t},
$$

where: $i=1,2, \ldots n_{n}, n_{n}$ is the number of elements in which the area in question was divided, $R_{t h A i}$ is the thermal resistance from the heat transferred from the external surface of the element $i$, and $R_{t h i j}$ is the thermal resistance for heat flowing from the node $j$ to the node $i$. The summation in Eq. (11) is carried out for all $j$ that are neighbouring element $i$. For the boundary condition of the first type, $\vartheta_{A i}$ is the known boundary temperature of element $i$. For the boundary condition of the third type, $\vartheta_{A i}$ is the temperature of the fluid beside element $i$.

The general form of the dependence that allows calculation of the thermal resistance values results directly from the heat conductivity Fourier law:

$$
R_{t h i j}=\int_{x_{i}}^{x_{j}} \frac{\mathrm{d} x}{\lambda_{i j}(x) A(x)},
$$

where: $x_{i}, x_{j}$ are the coordinates of the nodes $i, j$, respectively, and $A(x)$ is the surface area perpendicular to the integration path, along the line which joins the nodes.

Thermal resistance is related to heat transfer and can be obtained from the dependence: 


$$
R_{\text {thAi }}=\frac{1}{\alpha_{i} A_{i}},
$$

where: $\alpha_{i}$ is the heat transfer coefficient and $A_{i}$ is the surface area of element $i$.

The resulting system of ordinary differential equations shown in Eq. (11) describes the transient thermal field in the area in question. For the zero boundary condition of the second type $R_{t h A i} \rightarrow \infty$.

Within the rotor core, the volume density of heat sources is the quotient of the iron loss $P_{F e r}$ and its volume $V_{F e r}$ :

$$
q_{V F e i}=\frac{P_{F e \mathrm{r}}}{V_{F e \mathrm{r}}},
$$

Within the bar and ring area of the soldered squirrel cages the volume density of heat sources is:

$$
q_{V i}=J_{i}^{2} \rho_{R i},
$$

where: $J_{i}$ is the current density in the element $i$ and $\rho_{R i}$ is the resistivity of the material of element $i$. For the relatively large width end rings located far from the core, it can be assumed that the current density is of uniform nature, or if otherwise, the density distribution in ring section should be taken into consideration. By assuming uniform density, underrated temperature values are obtained in some areas of the end ring.

Concerning the motor with a cast squirrel-cage, the rotor impedance and current density distribution can be calculated with the conductor element method [6]. The method assumes that the bar fitted into the slot is composed of insulated conductors, the sections of which are matched to individual sections of the slot that are then joined together outside the core. The current displacement effect is neglected separately in each conductor. Fig. 4 shows the division of the rotor bar into pa modules. The equivalent circuit of the $k$ module is presented in Fig. 5.

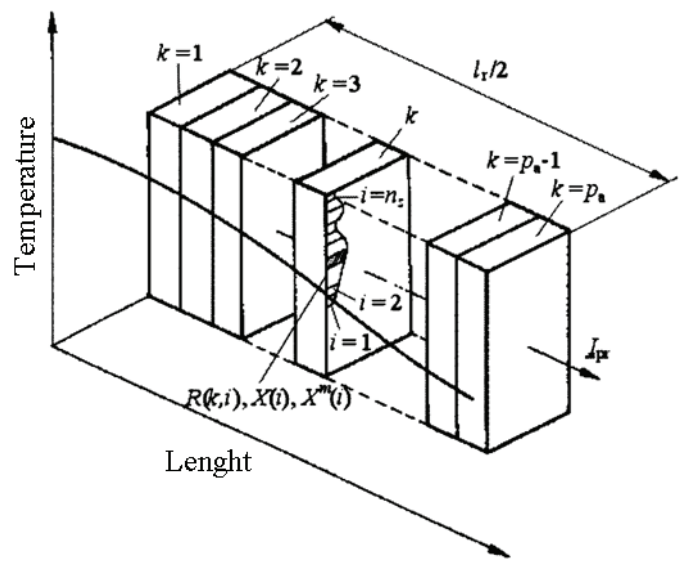

Fig. 4. Modules in the rotor bar 
By connecting the circuits (obtained for each of the $p_{a}$ modules) in series from Fig. 5, the equivalent circuit diagram for the entire bar is obtained, which is included in the whole equivalent circuit of the motor. Calculating the currents based on the equivalent diagram is done according to the algorithm presented in [6].

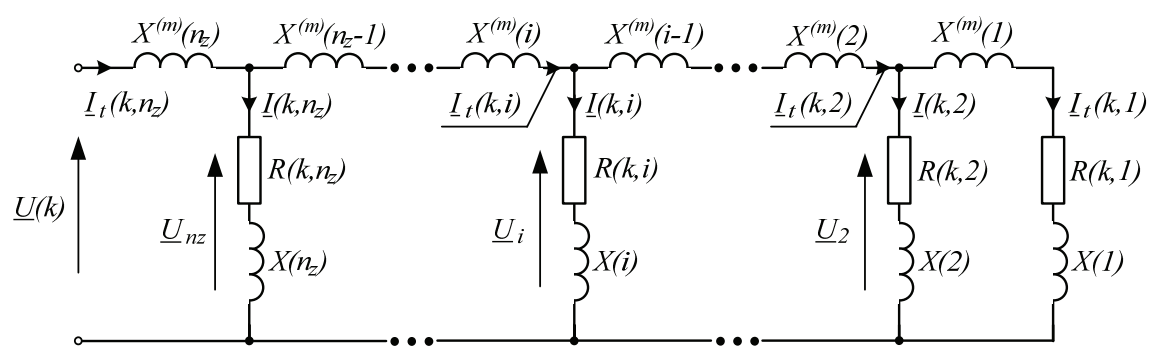

Fig. 5. Equivalent circuit of module $k$

The distribution of current density upwards in the bar in each module will be different because of the expected temperature changes along the motor axis, as shown in Fig. 4 . The current density in component $i$ of module $k$ is expressed in the dependence:

$$
J(k, i)=\frac{6 I(k, i) N_{s} k_{w s}}{A_{i} Q_{r} k_{s q}},
$$

where: $I(k, i)$ is the rms current $I(k, i)$ (Fig. 5), $k_{s q}$ is the skew factor, $k_{w s}$ is the stator winding coefficient, $Q_{r}$ is the number of rotor slots, and $N_{s}$ is the number of serial coils in stator phase winding. Hence the volume density of heat sources in component $i$ of module $k$ can be written as:

$$
q_{V i}=J^{2}(k, i) \rho_{R}\left[1+\alpha_{t h R} \Delta \vartheta(k, i)\right]
$$

where: $\Delta \vartheta(k, i)$ is the temperature increase of element $i$ in module $k$ above the ambient temperature, $\alpha_{t h R}$ is the temperature coefficient of resistance increase, $\rho_{R}$ is the resistivity of the material in ambient temperature.

Homogenous current density in the end ring is given as:

$$
J_{\mathrm{er}}=\frac{6 I_{t}\left(1, n_{z}\right) N_{s} k_{w s}}{2 A_{e r} Q_{r} k_{s q} \sin \frac{\pi p}{Q_{r}}},
$$

where: $I_{t}\left(1, n_{z}\right)$ is the rms total bar current $\underline{I}_{t}\left(1, n_{z}\right), A_{e r}$ is the surface area of the end ring, $p$ is the pole number. It is also assumed that the resistance change of individual ring components has no effect on the distribution of current density within the end ring. Good adhesion of cast bars to the sheet pack causes the cross-currents to occur, which are then contained in the pack, leading to the reduction of the ring current. That effect was not taken into consideration. 


\section{Measurement and calculation results}

Calculations of motor heating are done on the following sequence: switch on with the rotor blocked - switch off to cool down - start up. The calculations were performed using programs created by the author by means of Matlab software.

The calculations were performed on both a double-cage motor type SZDC196Ex with $320 \mathrm{~kW}, 6000 \mathrm{~V}$, and $p=3$ with a soldered squirrel-cage and on a cast cage type SCgx280M4 with $200 \mathrm{~kW}, 380 \mathrm{~V}$, and $p=2$. The measurements and calculations of the cage winding temperature supplied with a blocked rotor have been taken for the motor type SGR315M4 with $200 \mathrm{~kW}, 1000 \mathrm{~V}$, and a rated speed of $1479 \mathrm{r} / \mathrm{min}$, with a soldered cage. Table 1 shows the chosen parameters of the examined motors.

Table 1. Chosen parameters of the motors

\begin{tabular}{l|c|c|c}
\hline \multicolumn{1}{c|}{ Parameter } & $\begin{array}{c}\text { SZDC196Ex } \\
\text { (Motor A) }\end{array}$ & $\begin{array}{c}\text { SCgx280M4 } \\
\text { (Motor B) }\end{array}$ & $\begin{array}{c}\text { SCR315M4 } \\
\text { (Motor C) }\end{array}$ \\
\hline Rated power, $\mathrm{kW}$ & 320 & 200 & 200 \\
\hline Rated voltage, $\mathrm{V}$ & 6000 & 380 & 1000 \\
\hline Rated frequency, Hz & 50 & 50 & 50 \\
\hline Pole pair number & 3 & 2 & 2 \\
\hline Air gap, mm & 1.6 & 0.9 & 1.2 \\
\hline Stator diameter, m & $0.888 / 0.600$ & $0.470 / 0.308$ & $0.520 / 0.334$ \\
\hline Rotor diameter, m & $0.597 / 0.369$ & $0.306 / 0.105$ & $0.3316 / 0.130$ \\
\hline Core length, m & 0.56 & 0.31 & 0.35 \\
\hline Number of stator/rotor slots & $72 / 58$ & $48 / 38$ & $48 / 38$ \\
\hline Class of insulation & $\mathrm{F}$ & $\mathrm{F}$ & $\mathrm{F}$ \\
\hline Ratio of coil span to pole pitch & $10 / 12$ & $10 / 12$ & $10 / 12$ \\
\hline Number of stator-winding turns per coil & 240 & 44 & 56 \\
\hline Stator-winding resistance, $\Omega$ & 0.6479 & 0.0307 & 0.0369 \\
\hline
\end{tabular}

The duration of blocked rotor mode has been selected so that the maximum admissible cage temperature can be reached. The non-voltage interval has been selected to allow cooling of the hottest component of the cage to one third of the maximum admissible temperature. For squirrel cages with bars, the admissible temperature is approx. $350^{\circ} \mathrm{C}$ and $250^{\circ} \mathrm{C}$ for the cast cage [2]. After cooling, the motor was started up. It has been assumed that during the entire start up phase, the motor is subjected to constant torque that equals the maximum rated torque of the motor. The moment of inertia in both cases is selected to prevent thermal risks to the squirrel-cage. Fig. 6 presents the temperature changes calculated in selected locations (according to Fig. 3) of the motor (Motor B) with a cast cage, in the analysed operating mode. In Fig. 7, these form soldered cages (Motor A) are presented. Fig. 8 shows the thermal field in the slot axis of the motor with a cast cage (Motor B) in the all phases of the process calculated, i.e., operating mode with a blocked rotor, the non-voltage interval and the start up of the heated motor. The temperature of the starting cage of the motor with a soldered squirrel-cage (Motor A) varies as shown in Fig. 9. 


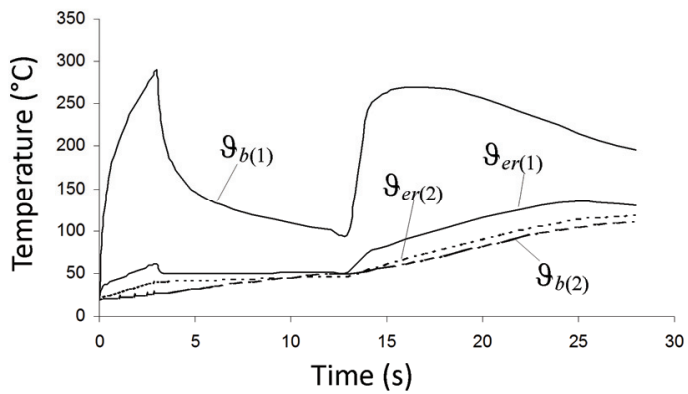

Fig. 6. Temperature changes in individual locations of the cast cage during motor blockage (3 s), non-voltage interval (10 s), and start up (15 s) (marking as in Fig. 3)

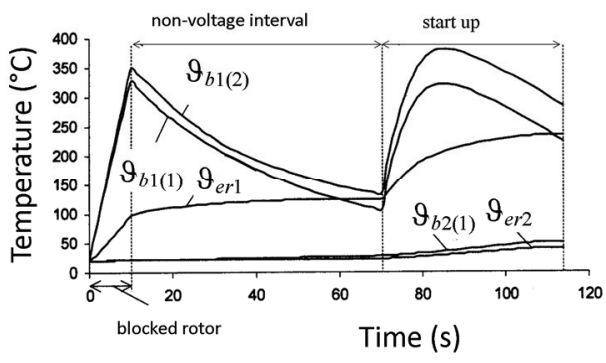

Fig. 7. Temperature changes in individual locations of the soldered cage during motor A blockage $(10 \mathrm{~s})$, non-voltage interval $(60 \mathrm{~s})$, and start up (45 s) (marking as in Fig. 3)

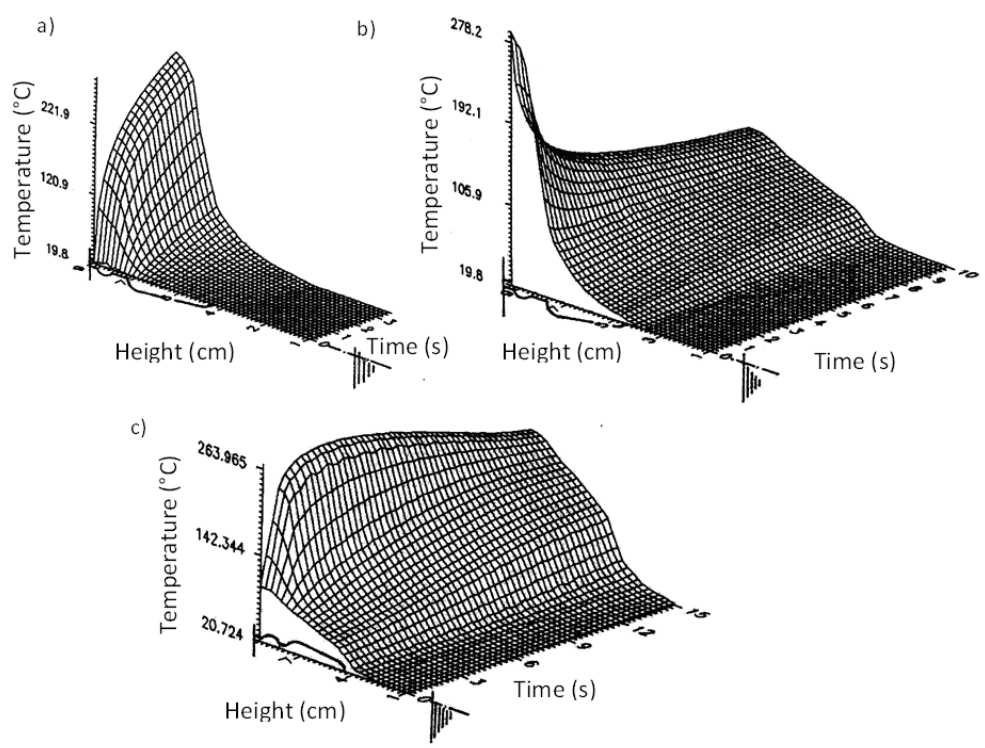

Fig. 8. Temperature changes in the slot axis in the motor with a cast cage (Motor B): a) while powered on with a blocked rotor; b) during cool down phase; c) during the start up phase

In the case of the motor with a bar cage (Motor A), the relationship between the times with a blocked rotor until the non-voltage interval, and until the start up (taking into consideration the above assumptions) is $10: 60: 40$ (Fig. 7). For the motor with a cast cage (Motor B), the relation is $3: 10: 15$ (Fig. 6). It can be concluded that the motor with the cast cage is less 
resistant to operating with a blocked rotor than the one with the bar cage. Accelerated heating of the cast cage in the top of the slot is a result of the strong current displacement. One should note that the lower winding parts in the slot reach considerably lower temperatures (Fig. 6).
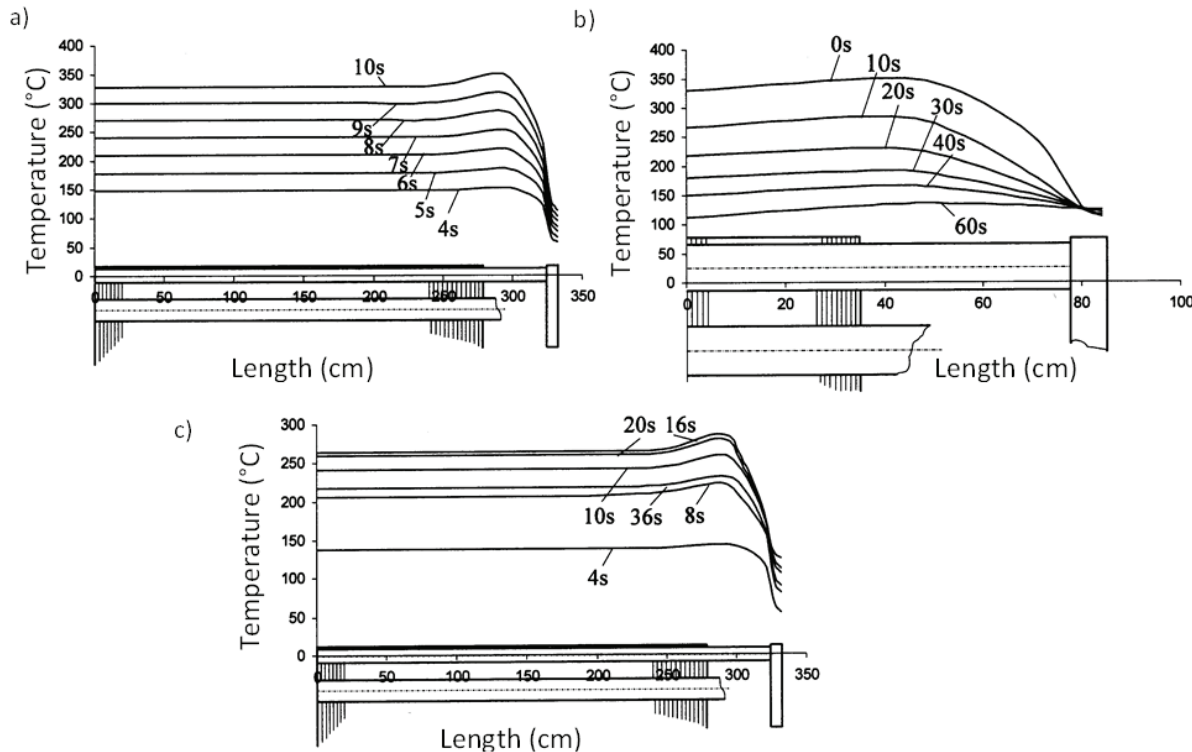

Fig. 9. Temperature distribution in the starting cage bar (Motor A): a) with power on with a blocked rotor; b) during cool down phase; c) during motor A start up

The time relations presented indicate that very hot parts of the cast cage cool down very quickly compared to the bar cage. Giving up the heat from the starting bars of the soldered cage to the rotor pack is difficult (slot clearance) and takes place mainly in the axial direction (towards the end rings), proven by the increase in the ring temperature $\vartheta_{e r 1}$ during the nonvoltage interval (Fig. 7). The heat from the hottest parts of the cast cage is additionally given up towards the slot bottom, and the temperature of components located there increases during the non-voltage interval (Fig. 6 and Fig. 8). The heated motor with the bar cage is capable of performing considerably longer start ups than that of the one with the cast cage. When the temperature of the hottest cage components in the operating mode in question reaches its limiting value, the temperature of the stator winding is far below the upper limit [2].

The measurements of cage winding temperature have been taken for the motor type SGR315M4 with a soldered cage (Motor C) with a rated power of $200 \mathrm{~kW}$, supply voltage of $1000 \mathrm{~V}$, with the rotor blocked. The ambient temperature during measurements was $\vartheta_{a}=30.5^{\circ} \mathrm{C}$, and the temperature inside the motor as read from thermocouples was $\vartheta_{0}=27^{\circ} \mathrm{C}$. The temperatures for the starting cage bar, the end region $\left(\vartheta_{b 1(2)}\right.$ in Fig. 3), and the end ring $\left(\vartheta_{e r 1}\right.$ in Fig. 3) were recorded while the motor was supplied with $968 \mathrm{~V}$ for $5 \mathrm{~s}$. 
Fig. 10 presents the measured and calculated temperature changes in the starting cage bar and ring in points $\vartheta_{b 1(2)}$ and $\vartheta_{e r 1}$ marked in Fig. 3 and in analogous points placed in the neighbouring slot pitch.

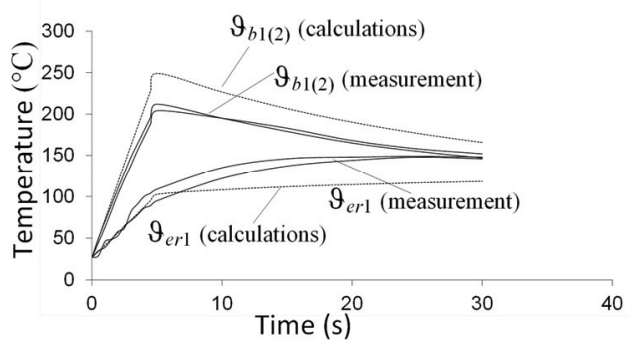

Fig. 10. Measured and calculated temperature changes in the starting cage bar (Motor $\mathrm{C}-$ see Table 1)

From the results presented in Fig. 10 it can be concluded that the differences between the calculated and measured values do not exceed $17 \%$. Several basic sources of such differences can be identified: inaccuracies resulting from thermocouples inertia and calibration of recording equipment, errors in determining the model parameters (heat transfer coefficients, slot clearance, etc.), inaccuracies in calculation sources of loos, asymmetry of rotor currents resulting from differences in positioning of the rotor slots in relation to the stator, and uneven adhesion of individual bars to slot walls.

\section{Conclusions}

The need to operate motors with a blocked rotor emerges occasionally (e.g., when the mechanical coal miner or coal mill is fully charged). Prolonged start ups resulting from large inertia pose serious risks to motor performance. Winding overheating and the occurrence of mechanical stress, resulting from increased temperature as a result of prolonged start up, also poses serious risk to the motor squirrel-cage. In the operating conditions referred to herein, the electromagnetic, thermal, and mechanical states are correlated.

Determining thermal fields in the winding of a double-squirrel-cage rotor is of great importance in those states because this is when there is a risk of shortening the life of the cage winding, as the stator temperature does not exceed the maximum values [2]. By using the double-squirrel-cage soldered winding, large values of starting torques are achieved; this is, however, at the cost of non-uniform heating of the cage, along with the tendency of the cage bars to overheat. Compared to a motor with a soldered cage, a motor with a cast cage is less resistant to operation with a blocked rotor or prolonged start up. However, the faster cooling of the winding compared to the bar cage is an advantage while operating the motor with frequent shorter start ups.

Carrying out experimental research on high-power motors involves a range of logistic operations as it usually has to be carried out in industrial conditions. It generates a high cost, which explains why a relatively small number of publications is currently being devoted to 
experimental research on heating problems in high-power squirrel cage induction motors [7-10]. This type of research is generally limited to steady-state operations.

The model can also be used by the motor user to establish the maximum admissible start up time, the operating time with blocked rotor, the length of the interval between the subsequent start ups, and the admissible number of start ups, as numerous systems protecting the motor from faulty start up require estimation of such settings.

The theoretical solutions presented herein have been verified only partially by the measurements described. The results obtained indicate that there is a particular need to examine the observed effects related to double-squirrel-cage motors. Thermal aspects of difficult start ups are of primary importance in terms of drive reliability because, regardless of the chosen start up method, unfavourable deformations occur to the heated cage components, which are then accompanied by detrimental mechanical stress. Due to this fact the design of a double squirrel cage induction motor is still being perfected [11].

\section{References}

[1] Chan C.C., Yan L., Chen P., Wang Z., Chau K.T., Analysis of electromagnetic and thermal fields for induction motors during starting, IEEE Transactions on Energy Conversion, vol. 9, no. 1, pp. 53-60 (1994).

[2] Mróz J., The analysis of coupled electromechanical and thermal problems in transient states of double-cage induction motor, Oficyna Wydawnicza Politechniki Rzeszowskiej (in Polish), Rzeszów (2013).

[3] Feyzi M.R., Parker A.M., Heating in deep-bar rotor cage, IEE Proceedings - Electric Power Applications, vol. 144, no. 4, pp. 271-276 (1997).

[4] Runge B., Berchnung der Stillstandserwarmung explosionsgeschutzter Asynchronmotoren mittels dreidimensionaler Finite-Elemente-Methode und Zeitschrittrechnung, Electrical Engineering, vol. 79, no. 3, pp. 179-191 (1996).

[5] Taler J., Duda P., Solving straight and reverse problems of heat conduction, WNT (in Polish), Warszawa (2003).

[6] Śliwiński T., Methods of calculating induction motors, Analysis (in Polish), PWN, Warszawa, vol. 1 (2008).

[7] Krok R., Influence of work environment on thermal state of electric mine motors, Archives of Electrical Engineering, vol. 60, no. 3, pp. 357-370 (2011).

[8] Al'Akayshee Q., Staton D.A., $1150 \mathrm{hp}$ motor design, electromagnetic and thermal analysis, ICEM-15-th International conference on electrical machines, Bruges, Belgium (2002).

[9] Dymond J.H., Ong R., Stranges N., Instrumentation, Testing, and Analysis of Electric Machine Rotor Steady-State Heating, IEEE Transactions on Industry Applications, vol. 38, no. 6, pp. 1661-1667 (2002).

[10] Zhang Y., Ruan J., Huang T., Yang X., Zhu H., Yang G., Calculation of Temperature Rise in Aircooled Induction Motors Through 3-D Coupled Electromagnetic Fluid-Dynamical and Thermal Finite-Element Analysis, IEEE Transactions on Magnetics, vol. 48, no. 2, pp. 1047-1050 (2012).

[11] Poprawski W., Wolnik T., Innovative design of double squirrel cage induction motor for high start frequency operation, Maszyny Elektryczne - Zeszyty Problemowe (in Polish), vol. 111, no. 3, pp. 41-44 (2016). 